\title{
Special focus on analysis and control of finite-valued network systems*
}

Finite-valued network system (FVNS) is a kind of network systems whose state, input and output take values from a finite set. FVNS has wide applications in the research fields such as systems biology, game theory, digital circuits, finite automata, graph theory, fuzzy control, nonlinear feedback shift register, and so on. Recently, an algebraic state space representation (ASSR) approach has been developed for the analysis and control of FVNS, which can convert an FVNS into a linear-like system. The ASSR based investigation of FVNS has attracted many scholars' research interests in the last decade.

This special focus of SCIENCE CHINA Information Sciences is devoted to covering the recent advances in finite game, residual gas fraction in IC engines, feedback shift register and petri net for FVNS. We have accepted five contributed papers with novel results that are established by the research groups in the field as follows:

"From STP to game-based control" provides a comprehensive survey on semi-tensor product (STP) of matrices and its applications to different disciplines. It covers some basic properties of STP as well as applications of STP to conventional dynamic systems, logical systems and finite games.

In "A survey on applications of semi-tensor product method in engineering", a comprehensive survey on the applications of STP method in many engineering fields such as gene regulation, power system, wireless communication, smart grid, information security, combustion engine and vehicle control are devoted. Some potential applications of STP method in engineering are also provided.

"Logical control scheme with real-time statistical learning for residual gas fraction in IC engines" develops an optimal control scheme for reducing the fluctuation of residual gas fraction (RGF) under variational operating condition by combining stochastic logical system approach with statistical learning method.

In "Nonsingularity of Grain-like cascade FSRs via semi-tensor product", the Grain-like cascade FSRs are converted into an equivalent linear equation based on STP. It regards the Grain-like cascade FSRs as Boolean control networks and presents some sufficient and necessary conditions of their nonsingularity.

"Modeling and analysis of colored petri net based on the semi-tensor product of matrices" establishes the marking evolution equation for colored petri net by using STP. It presents some necessary and sufficient conditions of reachability and controllability for colored petri net.

Finally, we would like to express our sincere appreciation to all the authors for submitting their manuscripts. Moreover, we express our deep gratitude to all the anonymous reviewers for delivering

\footnotetext{
${ }^{*}$ Citation Li H T, Shen T L, Lu J Q. Special focus on analysis and control of finite-valued network systems. Sci China Inf Sci, 2018, 61(1): 010200, https://doi.org/10.1007/s11432-017-9300-8
} 
high-quality and timely review comments. We also thank SCIENCE CHINA Information Sciences Editorial Office for their professional services and supports during the whole process of this special focus.

Guest Editors:

Haitao LI

Shandong Normal University, China

Tielong SHEN

Sophia University, Japan

Jianquan LU

Southeast University, China 